\title{
The Optical Design of the Soft X-ray Self Seeding at LCLS
}

\author{
Daniele Cocco ${ }^{*}$, RafaelAbela ${ }^{\mathrm{b}}$, John W. Amann ${ }^{\mathrm{a}}$, KenChow ${ }^{\mathrm{c}}$, Paul J. Emma ${ }^{\mathrm{c}}$, Yiping Feng ${ }^{\mathrm{a}}$, Georg \\ L.Gassner ${ }^{\mathrm{a}}$, Jerome Hastings ${ }^{\mathrm{a}}$, Philip Heimann ${ }^{\mathrm{a}}$, Zhirong Huang ${ }^{\mathrm{a}}$, Henrik Loos ${ }^{\mathrm{a}}$, Paul A. Montanez ${ }^{\mathrm{a}}$, \\ Daniel Morton ${ }^{\mathrm{a}}$, Heinz-Dieter Nuhn ${ }^{\mathrm{a}}$, Daniel F.Ratner ${ }^{\mathrm{a}}$, Larry N. Rodes ${ }^{\mathrm{c}}$, Uwe Flechsig ${ }^{\mathrm{b}}$, James

$$
\text { J.Welch }{ }^{\mathrm{a}} \text {, Juhao } \mathrm{Wu}^{\mathrm{a}}
$$

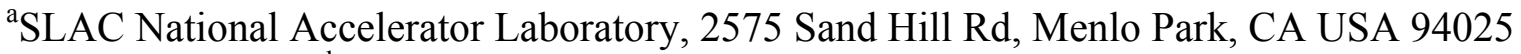 \\ ${ }^{\mathrm{b}}$ PaulScherrerInstitut, 5232 Villigen PSISwitzerland \\ ${ }^{c}$ Lawrence Berkeley National Lab, 1 Cyclotron Road Berkeley, CA USA 94720
}

\begin{abstract}
After the successful demonstration of the hard X-ray self-seeding at LCLS ${ }^{1}$, an effort to build a system for working in the soft X-ray region is ongoing. The idea for self-seeding in the soft X-ray region by using a grating monochromator was first proposed by Feldhauset. $\mathrm{al}^{2}$. The concept places a grating monochromator in middle of the undulators and selects a narrow bandwidth "seed" from the SASE beam produced by the upstream section of undulators, which is then amplified to saturation in the downstream section of the undulators. The seeded FEL beam will have a narrower bandwidth approaching the transform limit. The challenge is to accommodate a monochromator and refocusing system as well as the electron beam magnetic chicane into a very limited space. The Soft X-raySelf Seeding system replaces only a single undulator section of $\sim 4 \mathrm{~m}$. Theoverall project and the expected FEL performances are described elsewhere ${ }^{3}$. Here we present the detailed optical design solution, consisting of a fixed incidence angle toroidal blazed grating with variable groove density, a rotating plane mirror (the only required motion for tuning the energy) to redirect the selected monochromatic beam onto an exit slit, and two more mirrors, one sphere and one flat, to focus and overlap the 'seed' onto the electron beam in the downstream undulators.
\end{abstract}

Keywords:Free Electron Laser, Self-Seeding,Variable Line Spacing Gratings, Soft X-ray monochromator

\section{INTRODUCTION}

In the past few years, FLASHin the UV and Soft X-ray (SXR) range and LCLS in the SXR and Hard X-ray (HXR) range, demonstrated the power of the ultra-intense and ultra-short radiation produced by a Free Electron Laser (FEL). In both cases, the radiation was produced by the Self-Amplified Spontaneous Emission (SASE) mechanism. The pulse energy produced by a SASE source is very intense and has a very short duration, allowing the realization of experimental techniques not possible with any other type of machine. However, SASE-produced radiation suffers poor shot-to-shot reproducibility. The centroid of the energy spectrum jitters, and both the spectral and time profiles are spiky.Moreover, the bandwidth of a SASE beam is of the order of $0.2-0.3 \%$ (or higher) of the fundamental emitted photon energy. These peculiarities do not preclude the execution of most of the experiments, but some of them need an extra monochromator to clean up the beam. To create an FEL beam with Gaussian profile, both in time and energy spectrum, a couple of approaches were suggested. One of them is direct seeding, where an external laser, emitting a particular wavelength $\lambda$, "seeds" the electron beam to create a coherent emission at the same wavelength $\lambda$, but with amplified pulse power. More recently, theFermi@Elettrateamemployed a technique called High Gain Harmonic Generation (HGHG). In this case, there is still an external laser seeding the electron beam at a particular wavelength $\lambda$, but the radiation is emitted at a shorter wavelength $\lambda / n^{4,5,6}$. In both direct seeding and HGHG, the maximum photon energy (or minimum photon wavelength) is limited by the availability of an external seeding laser with enough power and with proper temporal characteristics at short enough wavelength.

A way to take advantage of the seeded photon beam characteristics and overcome the limit of available maximum photon energy is the adoption of the Self Seeding scheme. Self-Seeding is a technique that can be implemented in SASE FELs to narrow the bandwidth of the emitted radiation whilepreserving the pulse energy and therefore increasing the brightness of the emitted pulses. Self Seedingwas proposed by Feldhaus at $\mathrm{al}^{2}$ back in 1997 and is based on thefollowing idea. First, a set of undulatorsproduces SASE radiation at a particular wavelength. This radiation is filtered by a 
monochromator thereby narrowing the bandwidth. The radiation exiting the monochromator acts as an "external" seed in a second series of undulators for the same bunch of electrons that produced the radiation. The energy resolution of the monochromator determines the bandwidth of the photon pulses exiting the second undulator chain,andthe energy spectrum will have an almost perfect Gaussian profile. A working prototype operating in the HXR region was installed at the Linac Coherent Light Source (LCLS) at the SLAC National Accelerator Laboratory ${ }^{1}$. This system, based on a single diamond crystal working in forward Bragg diffraction geometry, was designed to optimally work at $8.3 \mathrm{keV}$ photon energy. Due to the lattice characteristics of the usable crystals (mainly Diamond and Silicon) it can be implemented only for photon wavelengths well below $1 \mathrm{~nm}$, e.g. in the HXR and not in SXRHowever, the success of this first system brought us to push for the realization of a self-seeding scheme operating into the SXR region.

While in the HXR range a single crystal is sufficient to generate the self-seeding beam, a full SXR monochromator is much more complicated, involving a grating, a slit (optional),and focusing and/or refocusing optics. On top of that, this optical system must be hosted in a single undulator section length, sharing the already limited available space with all the components needed to properly handle the electron beam for reuse in the downstream undulator chain. This requirement is driven by the necessity to preserve as much undulator sections as possible for the normal SASE operational mode

Practically, one needs to separate the electrons that have produced the SASE beam from the photon trajectory. Once this is done (by using a series of deflecting magnets called a chicane)the monochromator selects a narrow bandwidth of the photon beam and refocuses the monochromatized beam at the proper photon energy into the following undulator. The electrons are re-deflected to have again the same trajectory of the photons. To achieve temporal overlap, the electron chicane must delay the electrons the same amount of time that the monochromator system delays the photon beam.

The complexity of the problems associated with all the constraints and requirements made the SXR Self Seeding a theoretically very interesting system, but a very challenging instrument, so that until now it was never been adopted. At LCLS we have found a very compact solution fitting into a single undulator section, providing variable energy from 500 to $1000 \mathrm{eV}$ (with potential to eventually reach 300-1200 eV)by using a single toroidal grating and a single movement (a mirror rotation) to perform the photon energy scan. This system is under construction and will be installed in the fall of 2013, with commissioning beginning soon after. The details of the optical design and the expected performance will be reported in the following chapters.

\section{SYSTEM AND OPTICAL DESIGN}

The SXR Self Seeding (SXRSS) system (shown in figure 1) is schematically composed of the following three components:

- The four-dipole magnetic chicane; to deflect the electron beam out of the trajectory of the SASE photons, to delay the electron bunch of the proper amount to be temporally coincident with the monochromatic photon beam, and to spatially overlap the electrons, after the monochromator, with the photon beam, and to wash out the microbunching accumulating in the preceding undulator section.

- The Grating based monochromator; to select the proper photon energy and monochromatise (reduce the energy bandwidth) of the SASE beam

- Diagnostic system; to measure the overlap of the electrons with the photons after the monochromator (called BOD, Beam Overlap Diagnostic) 


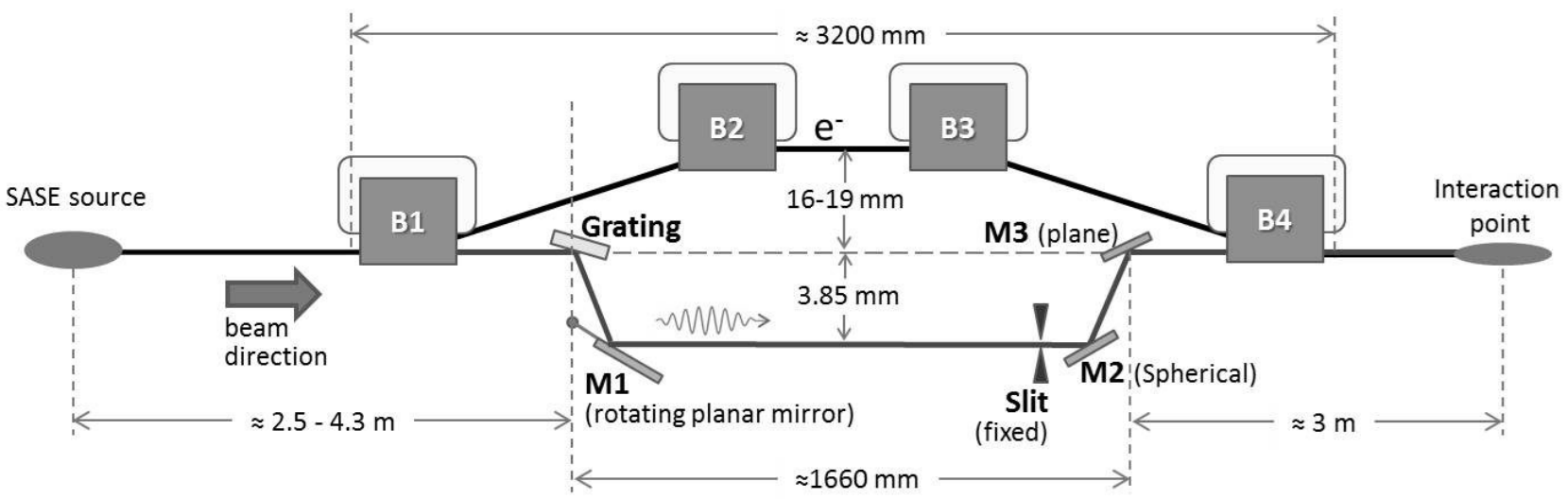

Figure 1: Top view of the SXRSS system. The optical system is composed by (from left to right) a toroidal grating, a rotating plane mirror, an energy selective slit, a focusing spherical mirror and a plane mirror to redirect the beam in the proper direction. The four magnets (B1-B4) deflect the electrons away from the optical devices.

The principal requirements for the SXRSS are:

- Photon energy range 500-1000 eV

- Have a resolving power $(\mathrm{E} / \Delta \mathrm{E}) \geq 5000$

- Produce a nearly transform limited pulse

- Induce a delay of the x-ray (with respect a non-deflected beam) lower than $1 \mathrm{ps}$

- Produce an image of the source in the interaction point (nearly located in the center of the following undulator) just slightly larger than the electron beam in the same location

- To be fully removable to allow normal SASE operation.

- To fit within one undulator section $(<4 \mathrm{~m})$

- To have a stay clear distance of $2 \mathrm{~mm}$ or more from the electron beam

- To transmit enough power to seed the electrons in the following undulator (estimated to be $20 \mathrm{~kW}$ or more)

- To provide steering capability to overlap the electron and the X-ray beam downstream (by using the BOD as feedback)

The topmostchallenges encountered in the design of this system were the very limited space available, the need to share the space, and the vacuum chambers, with the magnetic chicane system, and the need to work in a radiation area, e.g. no easy access to the system zone. The first of these constraints poseda serious challenge. For instance, a Self-Seeding monochromator design as proposed by R. Reininger ${ }^{7,8}$ for Flash, in Hamburg, cannot be adopted because of the length. Another proposed solution, explicitly for LCLS ${ }^{9}$, even if optically perfect, could not be implemented because of interferences with the needed magnetic chicane hardware. Nevertheless, the optical design adopted and described in the following subsection is based on it.

\subsubsection{Optical design}

The design of the optical system, as explained above, was mainly driven by the mechanical and space constrain. Accommodating translations and rotations in such a limited space and so close to magnets and electron trajectory, is quite a problem. Therefore, beside the need to save space, we also needed to reduce to a minimum the range of movements. To satisfy all the requirements listed above we came out with the design shown in figure 1.

The central two magnets of the chicane wereplacedtogether as close as possible to leave as much room as possible to the optics. Nevertheless, even if this solution gives the maximum available transverse and longitudinal space for the optics, it also leaves just two positions to install the optics. In the first break (between magnets B1 and B2), we positioned the fixed incidence angle toroidal Variable Line Space (VLS) grating. The VLS toroidal grating disperses and focuses the 
radiation of different wavelengths at different directions but almost at the same focal distance (at least in the 500-1000 $\mathrm{eV}$ region). Therefore, just a rotating plane mirror (M1) is sufficient to select the proper wavelength and direct it in the exit slit, almost $1.3 \mathrm{~m}$ downstream. In the second position between the magnets B3 and B4, are the exit slit, the refocusing mirror, and a plane mirror to convey the beam in the proper direction.

Since the X-ray beam has to pass through the magnets vacuum chamberinside the small gap in B2 and B3, it was impossible to have the X-ray beam and the electron beam in two orthogonal planes. Moreover, the vacuum chambers have to be sharedby both the electron and X-ray beams (figure 2). This constraint together with the need to not induce too large a photon beam delay, forced us to have a monochromator dispersing in the horizontal plane with an offset between incoming and outgoing X-ray beams of $3.85 \mathrm{~mm}$ only - a factor 5 less than the usual configuration. The other difference with respect the standard monochromator is the fact that the grating is positioned before the rotating mirror and not vice versa. In this way, we can have a fixed first optics (e.g. fixed incidence angle grating) and a rotating second optics (the plane mirror) that is away from the electrons trajectory and therefore can be actuated safely (figure 3 ).

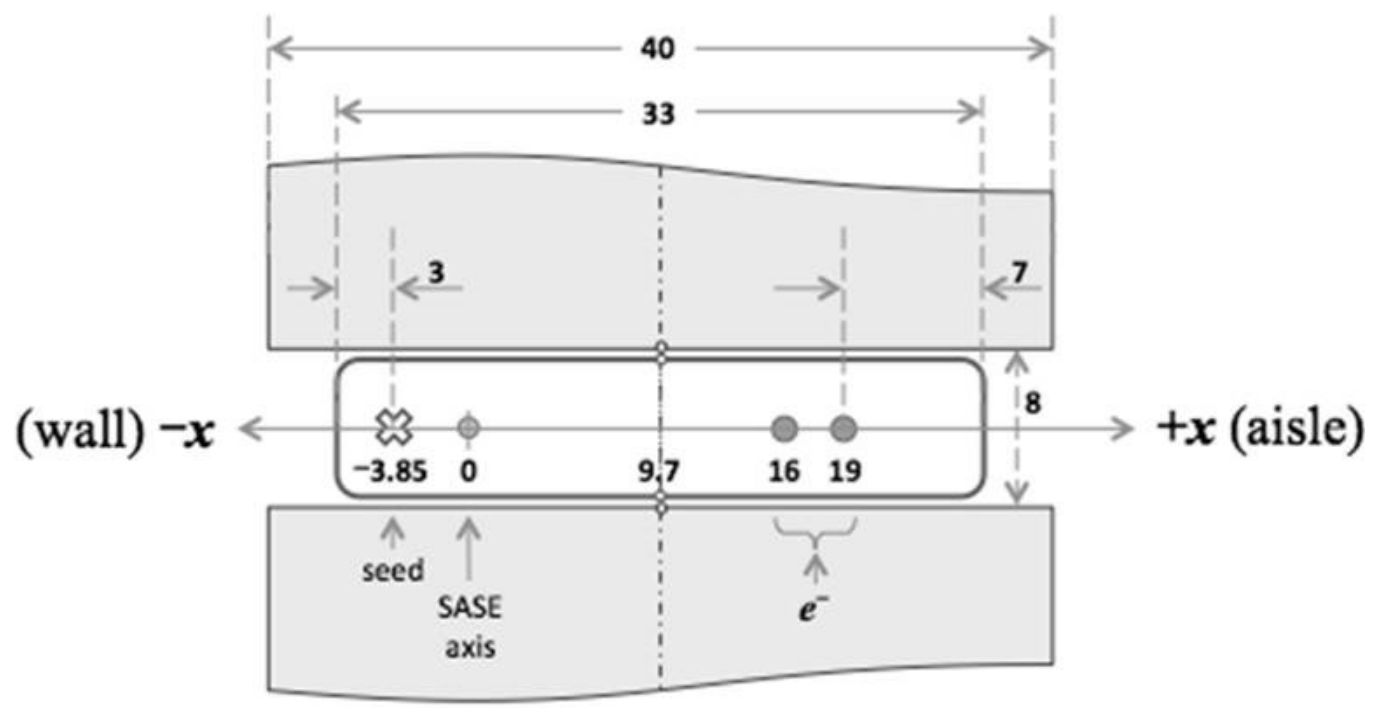

Figure 2: Position of the photons (left side of the picture) and electrons in the B2/B3 chicane vacuum chamber (for both minimum and maximum delay). Magnet pole centers are horizontally offsetby $9.7 \mathrm{~mm}$ toward the aisle with respect to the SASE axis. The $\mathrm{x}$-ray seed beam is $3 \mathrm{~mm}$ from the wall-side chamber wall ( $33 \mathrm{~mm}$ wide), and the extreme electron $\mathrm{x}$ position (maximum delay) is $7 \mathrm{~mm}$ from the aisle-side chamber wall.

\subsubsection{Monochromator design}

As already said, the monochromator is based on a VLStoroidal grating. The SASE radiation produced by the first 8 undulators, impinges on the grating with an $89^{\circ}$ fixed angle of incidence with respect the grating normal.The toroidal shape, together with the VLS pattern on the grating, lets one focus the beam in both directions. In the vertical or sagittal direction, the beam is refocused in the middle of the following undulator (the electron-photon interaction zone). In the horizontal (or tangential) direction, it focuses the radiation onto the exit slit plane (almost $1.3 \mathrm{~m}$ downstream of the grating).

Let us start considering the tangential direction. If we consider the angles of incidence and diffraction with respect the grating normal (as in figure 3), the grating equation describing the relation ofthe angle of incidence $\alpha$, the angle of diffraction $\beta$, and the grating groove density $\mathrm{D}$ (or grating groove spacing $\mathrm{d}=1 / \mathrm{D}$ ) is:

$$
\frac{n \lambda}{d}=\sin (\alpha)-\sin (\beta)
$$

From this equation, one can derive the direction of the diffracted beam. By orienting the plan mirror M1 at an angle equal to $(\alpha+\beta) / 2$ the beam is directed always in the same direction. This is the only needed motion of the whole system to select the proper wavelength. 


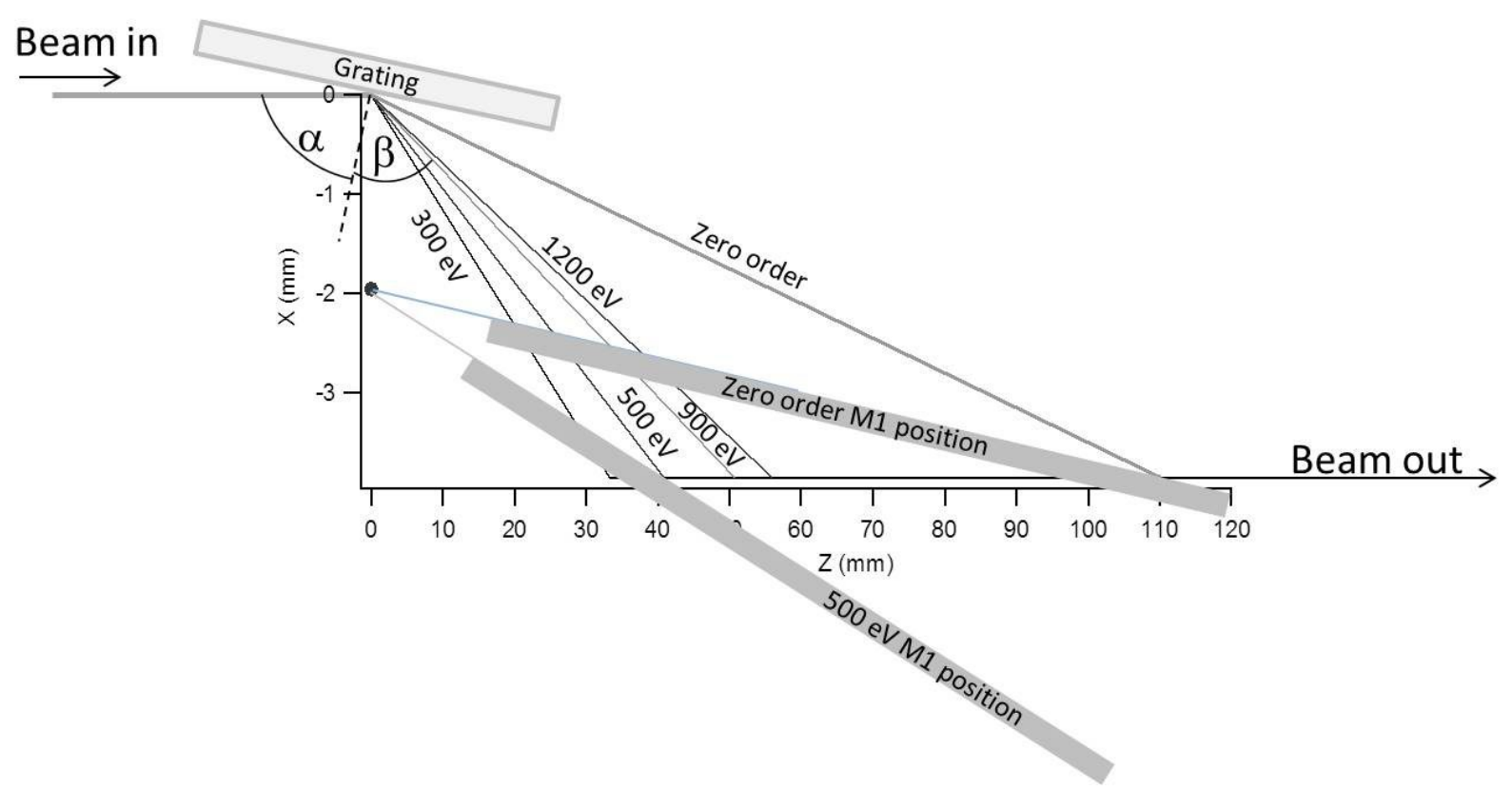

Figure 3: Relative position of the grating and the plane mirror M1 with some diffracted energy directions. Figure is not on scale

To calculate the focal property of a VLSgrating, one has to write the groove density D (lines $/ \mathrm{mm}$ ) as afunction of the position $x$ along the grating. The origin is the center of the grating and positive $x$ is toward the image.

$$
D(x)=D_{0}+D_{1} x+D_{2} x^{2}+\cdots
$$

The $\mathrm{D}_{0}$ parameter is the one used to calculate the diffraction direction in equation 1 . The second term of the polynomial series was usually used to reduce the aberration in a monochromator. Since $1998^{10}$ it was used to focus, or as a term to improve the focal property of the spherical grating ${ }^{11}$. In this design, it is used in a way similar to the second approach, i.e. to produce an almost constant focal distance for a fixed incidence angle spherical (or toroidal) grating.

By starting from the defocus equation $\mathrm{F}_{20}$ for a spherical substrate (eq. 3)

$$
F_{20}=-n \lambda D_{1}+\left(\frac{\cos ^{2} \alpha}{r}-\frac{\cos \alpha}{R}+\frac{\cos ^{2} \beta}{r^{\prime}}-\frac{\cos \beta}{R}\right)
$$

One can calculate the focal distance $r$ by zeroing equation 3, e.g. (eq. 4)

$$
r^{\prime}=\frac{\cos ^{2} \beta}{n \lambda D_{1}+\frac{1}{R}(\cos \alpha+\cos \beta)-\frac{\cos ^{2} \alpha}{r}}
$$

In equation 4 , one has to take into account that the source distance $r$, changes with the photon energy. By taking into account this effect and the available space in between of the magnets, $D_{1}$ was selected to be $1.61 / \mathrm{mm}^{2}$ and the radius of curvature $\mathrm{R}$ was chosen to be $195 \mathrm{~m}$. This selection does not provide a perfect fixed focal distance for the grating as a function of the energy (table 1) but the variation is almost negligible. In fact, a variation of $1 \mathrm{~mm}$ in the focal distance increases the spot by much less than $1 \mu \mathrm{m}$. Since the resolving power is diffraction limited (see next section) such spot enlargement does not influence the resolving power at all. The other possible problem could be the spot dimension at the re-entrance point (focusing position of mirror M2). A small change in longitudinal focal point or size is not a problem since the interaction length between electrons and photons is quite long (one gain length, e.g. 2-3 m). Therefore, even if the spot is focused a few $\mathrm{cm}$ earlier or later, it does not affect the seeding process. 


\subsubsection{Expected resolution}

The principal effects that determine the resolution in this system are the exit slit and the number of illuminated grooves of the grating.

The effect of the exit slit can be determined by the following equation (eq. 5)

$$
\frac{\lambda}{\Delta \lambda}=\frac{E}{\Delta E}=\frac{\lambda D_{0} r^{\prime}}{\cos \beta s^{\prime}}
$$

where s' is the monochromatic spot dimension at the exit slit and r' the grating focal distance.

The diffraction limited contribution is simply the number of grooves illuminated by the radiation, $\mathrm{N}(\mathrm{eq}, 6)$

$$
\frac{\lambda}{\Delta \lambda}=\frac{E}{\Delta E}=N=D w
$$

Since the source distance is quite small (table 1), the illuminated length (w) is also limited and therefore so is the number of illuminated grooves $\mathrm{N}$.

The independent effect of the two contributions is reported in table 1. As shown, the resolving power defined by the exit slit, with the ideal spot dimension shown in the same table, is a factor 2 larger than the diffraction limited contribution. Therefore, the latter will be the limiting factor. Nevertheless, resolving power in excess of 5000 is expected in most of the range.

\begin{tabular}{|c|c|c|c|c|c|}
\hline $\begin{array}{c}\text { Photon } \\
\text { energy } \\
(\mathrm{eV})\end{array}$ & $\begin{array}{c}\text { Source } \\
\text { distance } \\
(\mathbf{m m})\end{array}$ & $\begin{array}{c}\text { Resolving } \\
\text { power } \\
\text { Slit limited }\end{array}$ & $\begin{array}{c}\text { Resolving power } \\
\text { Diffraction } \\
\text { limited }\end{array}$ & $\begin{array}{l}\text { Grating focal } \\
\text { distance }\left(\mathbf{r}^{\prime}\right) \\
(\mathbf{m m})\end{array}$ & $\begin{array}{c}\text { Image size } \\
\left(\mathrm{s}^{\prime}\right) \\
(\mu \mathrm{m})\end{array}$ \\
\hline 500 & 2975 & 15538 & 6837 & 1349.0 & 3.16 \\
\hline 600 & 3211 & 14534 & 6150 & 1349.0 & 3.06 \\
\hline 700 & 3447 & 13931 & 5659 & 1349.2 & 2.95 \\
\hline 800 & 3683 & 13590 & 5290 & 1349.6 & 2.81 \\
\hline 900 & 3919 & 13438 & 5004 & 1350.1 & 2.67 \\
\hline 1000 & 4155 & 13434 & 4775 & 1350.8 & 2.52 \\
\hline
\end{tabular}

Table 1.General performance of the SXR SS monochromator as a function of the photon energy.

Since the resolving power is diffraction limited, the use of the exit slit may be not necessary. In fact, the mirror M2 reimages the exit slit onto the interaction point in the middle of the following undulator chain. The expected dimension of the photon beam in the interaction region is almost identical to the electron beam dimension over most of the photon energy range. If one does not use the exit slit, the M2 mirror does not only magnify the spot dimension but also the distance among the different energies. This means, practically, that if two particular energies were separated by a slit aperture s', they will be separated by an electron beam size at the interaction region. Since only the photons that interact with the electron contribute to the seeding process, the electrons act as an exit slit.

The exit slit is not mandatory but it could be anyway useful. First of all, in the alignment phase one has to be sure that the selected photon energy is properly aligned with respect the electron beam into the whole interaction region. Practically, by selecting a narrow bandwidth with the exit slit, one can align this "single" photon energy to be parallel and coincident with the electrons. If one starts without the exit slits, the information on the direction of a given photon energy is lost and the electrons could interact with different photon energy in different longitudinal positions. Moreover, even if the resolving power is dominated by the diffraction limit effect, there is still a small contribution due to the exit 
slit. If necessary, to increase the overall resolving power, one can use a narrow slit to reduce such contribution. Therefore, the exit slit is anyway suggested and, in this case, adopted.

\subsubsection{Design of the optics}

The electrons are deflected by the four magnets B1-B4 such that the induced delay, with respect the straight direction, matches the delay of the photon beam passing through the four optics. Such delay depends on the photon energy, since the rotating M1 mirror changes the total path traveled by the photons. The induced delay changes from $\sim 650$ fs for the highest energies to $800 \mathrm{fs}$ at $500 \mathrm{eV}$ and larger for lower energies.

The equivalent angles, induced by the magnets, range from 16 to almost 18 mrad. With such a small angle and considering the very short distance from the magnets B1 to the grating and from $\mathrm{M} 3$ to B4, the available space in the back of the optical surface is as small as $8 \mathrm{~mm}$. Moreover, the minimum safe distance from any obstacle to the electron beam is $2 \mathrm{~mm}$. This leaves a usable space of $\sim 6 \mathrm{~mm}$ for the mirror bulk (figure 4 ).

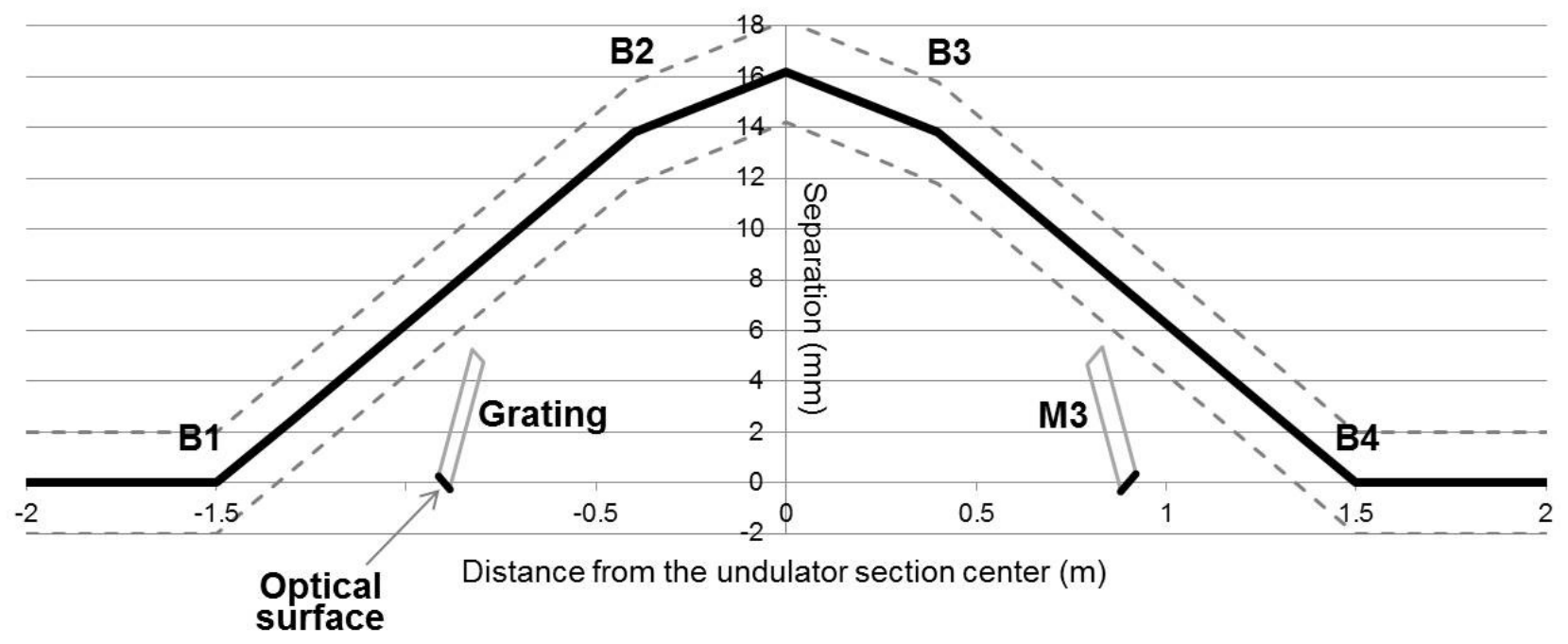

Figure 4: Stretched sketch of the separation between electrons and the two closest optics, the grating and M3. The electrons trajectory is represented by the black fat line. A $22 \mathrm{~mm}$ free area around is emphasized by the dashed grey lines. The mirror and grating thickness is supposed to be $5 \mathrm{~mm}$.

In principle the systems can be realized by using a $5 \mathrm{~mm}$ thick mirror, as shown in figure 4 . However, since the required slope and shape errors are quite tight, one would have to adopt a complicated mechanical holder to preserve such characteristics. Moreover, the edges of the optics are very close to the electron safety area. All such concerns bring us to pursuing a different solution.

The M3 and the grating are realized by polishing a much thicker substrate $(30 \mathrm{~mm})$. Once the proper optical figuring is obtained, a hole, centered $10 \mathrm{~mm}$ from the optical surface is drilled all along the tangential direction (figure 5). The hole diameter is $12 \mathrm{~mm}$ so that the hole starts $4 \mathrm{~mm}$ from the optical surface and there is enough room for the electrons to pass through the hole without being too close to the mirror bulk. Having the hole larger and closer to the surface would have introduced major deformation to the optical surface; therefore, the decision was a compromise between the need of clearance and the need of good optical surfaces. Anyway, such solution has the double advantage to give more room between the mirror bulk and the electrons and to have much easier to handle optical elements. 

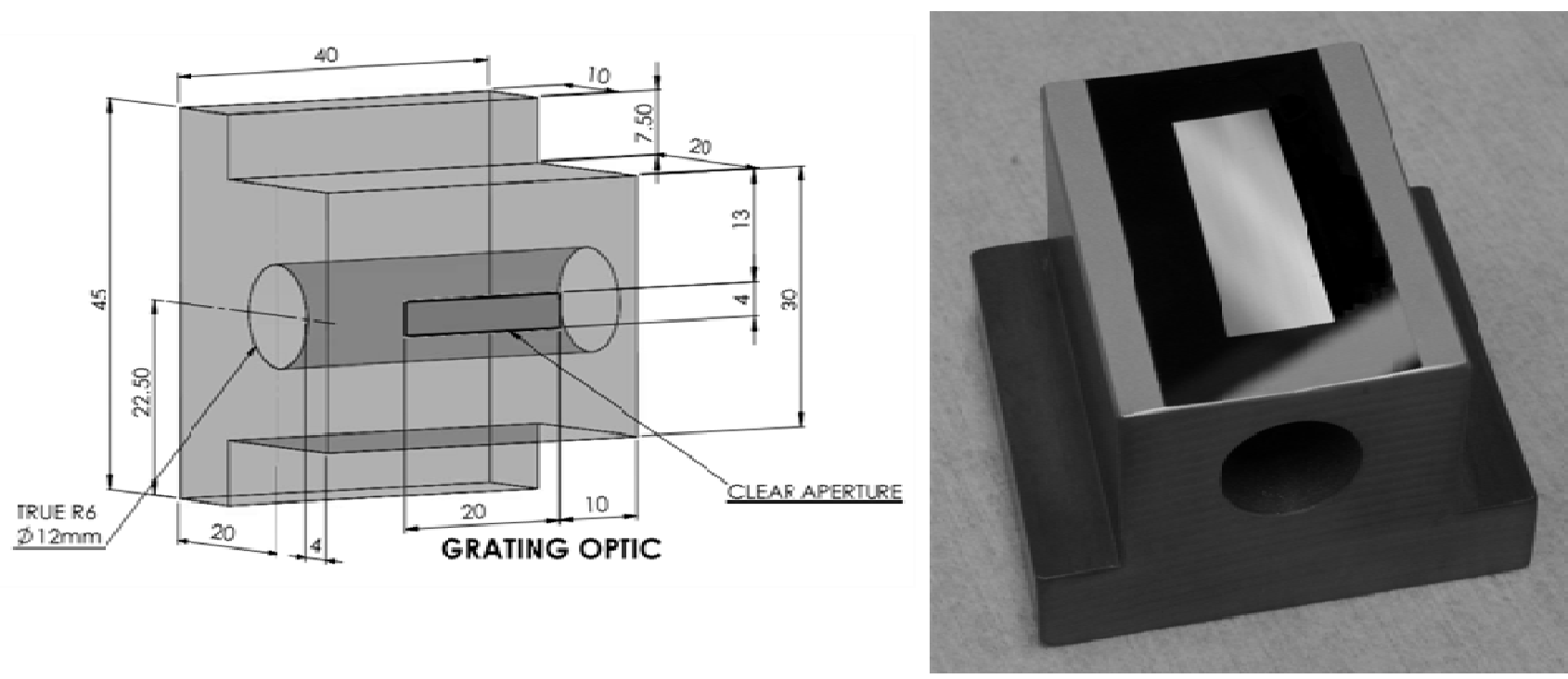

Figure 5: Dimensions (left) and picture (right) of the toroidal grating.

\section{CONCLUSIONS}

The Soft X-ray Self seeding optical design was presented. The system is under installation and will be commissioned in winter 2013-2014. The design is optimized to make optimal use of the available space without sacrificing the requirements. The challenging optical substrates were produced by Pilz Optics, Germany, the coatings $(\mathrm{Pt})$ are deposited by AXO Dresden and the grating ruling was made by DIOS GmbH (Bruno Nelles) and HZB, Germany. The metrology characterization was done at HZB (Frank Siewert) and at PSI (SibylleSpielmann-Jäggi).

\section{REFERENCES}

[1] Amann, J. et al. "Demonstration of self-seeding in a hard-X-ray free-electron laser" Nature Photonics 6, 693 (2012)

[2] Feldhaus, J. et al. "Possible application of X-ray optical elements for reducing the spectral bandwidth of an X-ray SASE FEL" Opt. Commun.140, 341-352 (1997).

[3] Feng, Y., et al.,"System design for Self-Seeding the LCLS at Soft X-Ray Energies" Proc. FEL 2012 conference, Nara, Japan (2012)

[4] Yu L. H. "Generation of intense uv radiation by subharmonically seeded single-pass free-electron lasers" Phys. Rev. A 44, 5178-5193 (1991)

[5] Allaria, E., Callegari, C., Cocco, D., Fawley, W, Kiskinova, M., Masciovecchio, C., Parmigiani, F., “The FERMI@Elettra free-electron-laser source for coherent X-ray physics: photon properties, beam transport system and applications" New J. Phys. 12, 075002 (2010).

[6] Allaria, E. et al. "Highly coherent and stable pulses from the FERMI seeded free-electron laser in the extreme ultraviolet" Nature Photonics NPHOT-2012-03-00327B

[7] Reininger, R., et al., "Wavefront propagation through the beamline designed for seeding the DESYXUV FEL" Nucl. Instrum. And Meth A Vol 467-468 (1) pp. 38

[8] Reininger R. et al. (Eds.), Synchrotron Radiation Instrumentation, Eleventh US National Conference, AIP Conference Proceedings 521, American Institute of Physics, Stanford, CA (1999), p. 458

[9] Feng, Y. et al. "Optics for self-seeding soft x-ray FEL undulators",2010 FEL conference, Malmo, Sweden, (2010).

[10] Cocco, D. et al. "MicrofocusingVLS grating-based beamline for advanced microscopy”.SPIE proc. Vol. 3767 p 271 279 (1999)

[11] Cocco D. et al."ComIXS on BACH: a compact soft x-ray spectrometer operating at Elettra”,AIP Conf. Proc. Vol. 705 p. 873 (2004)

[12] Flechsig U. et al. "The upgraded LTP-V at SLS”, Nucl.Intrum.And Meth. A 710, 0, pp. 13 (2013) 